\title{
ESTUDIO BIBLIOMÉTRICO DE LAS REVISTAS 3C EMPRESA, 3C TIC Y 3C TECNOLOGÍA
}

\section{BIBLIOMETRIC STUDY OF 3C EMPRESA, 3C TIC AND 3C TECNOLOGÍA JOURNALS}

Inés Poveda-Pastor ${ }^{1}$

1. Máster Universitario en Información y Comunicación Científica, Universidad de Granada. Graduada en Información y Documentación, Universitat de València. (España).E-mail: inpopas@correo.ugr.es

\section{Citación sugerida:}

Poveda-Pastor, I. (2017). Estudio bibliométrico de las revistas 3C Empresa, 3C TIC y 3C Tecnología. 3C Tecnología: glosas de innovación aplicadas a la pyme, 6(4), 64-73. DOI: <http://dx.doi.org/10.17993/3ctecno.2017.v6n4e24.64-73/>. 


\section{RESUMEN}

En el siguiente trabajo se realiza un estudio bibliométrico sobre las tres revistas de la Editorial 3Ciencias: 3C Empresa, 3C TIC y 3C Tecnología, con el objetivo de examinar y analizar las características de las publicaciones periódicas y los autores de la Editorial 3Ciencias y su desarrollo entre los años 2012 y 2016. Por un lado, se analiza la producción científica a partir del recuento de datos, y por otro lado, la autoría, estudiando el número de autores por revistas, la procedencia de los mismos, y finalmente, aplicando la Ley de Lotka. Para finalizar, se revelan las principales características de las publicaciones y se establece la evolución de las mimas.

\section{ABSTRACT}

In this work, a bibliometric study is carried out on the three journals of the Editorial 3Ciencias: 3C Empresa, 3C TIC and 3C Tecnología. The aim is to examine and analyze the characteristics of the journals and authors of the Editorial 3Ciencias and their development between the years 2012 and 2016. On the one hand, the scientific production is analyzed from the data counting. On the other hand, it is investigated the authorship, studying the number of authors by journals, the origin of the same, and finally, applying Lotka's Law. To end, the main characteristics of the publications are revealed and the evolution of the same is established.

\section{PALABRAS CLAVE}

Estudio bibliométrico, Bibliometría, Producción Científica, Editorial 3Ciencias.

\section{KEY WORDS}

Bibliometric study, Bibliometrics, Scientific Production, Editorial 3Ciencias. 


\section{INTRODUCCIÓN}

La Editorial Científica 3Ciencias es una editorial española de prospección internacional nacida en 2012 que publica trabajos fruto de la investigación científica en forma de monografías y publicaciones periódicas, ambas en formato electrónico. En la presente investigación se realiza un estudio bibliométrico sobre la segunda tipología, las revistas.

La Editorial 3Ciencias publica 3 revistas, a decir: 3c Empresa, 3c Tic y 3c Tecnología. La primera, cuyo título completo es 3c Empresa: Investigación y pensamiento crítico, abarca las ciencias económicas y empresariales. 3c TIC: Cuadernos de desarrollo aplicadas a las TIC, intersecciona la Información y la Comunicación, dos ciencias que agrupan las técnicas y elementos usados en el tratamiento y la transmisión de la información, principalmente se abarca la Informática, la Internet y las Comunicaciones. Finalmente, 3c Tecnología: Glosas de innovación aplicadas a la pyme, aborda el campo de la innovación tecnológica y la Ingeniería.

El objetivo del presente estudio es examinar y analizar las características de las publicaciones y los autores de la Editorial 3Ciencias y su desarrollo entre los años 2012 y 2016.

Para ello, se realiza un análisis bibliométrico, que es una combinación de diferentes métodos para llevar a cabo análisis cuantitativos de la ciencia (Zeleznik, et al., 2017). Se considera que este tipo de estudios constituyen actualmente herramientas muy empleadas para la evaluación de las publicaciones seriadas y científicas (Paz Enrique, et al. 2016). En este caso, se analiza primeramente la producción científica a través los artículos publicados por año y revista, contabilizando el número de artículos, y la media, moda y mediana de los mismos. Seguidamente, el estudio se centra en un análisis de autoría, pues se indican los autores por revista y el porcentaje de autores nacionales e internacionales además de la procedencia de los mismos. Finalmente, se aplica la Ley de Lotka de productividad para los autores de las revistas. Sin duda, es importante comprometerse con los usos de las métricas en muy diversos contextos, donde el empleo de indicadores bibliométricos podría tener grandes consecuencias para el investigador individual (Hammarfelt y Rushforth, 2017).

\section{METODOLOGÍA}

Tal y como señala Ardanuy (2012), para el análisis bibliométrico se necesita disponer de cuantiosa información bibliográfica. Es por ello que se ha hecho una extracción minuciosa de los datos requeridos para el presente estudio.

Para el tratamiento y manejo de los datos se ha utilizado el paquete office, en concreto la herramienta Excel, y se han extraído de los archivos de la revista de forma manual para así obtener los datos con la mayor precisión posible.

Todos los artículos que se han incluido en este trabajo están escritos en español, pues aunque se aceptan artículos en otras lenguas, el idioma que predomina es el español, y además todos ellos son artículos científicos originales. 


\section{RESULTADOS}

Como se ha mencionado anteriormente, los datos se extraen para analizar, primeramente la producción científica, basada en el recuento de las publicaciones, y en segundo lugar, la autoría, teniendo en cuenta número de autores por revista, procedencia de los mismos, y finalmente aplicar la Ley de Lotka.

\subsection{PRODUCCIÓN CIENTÍFICA}

En este primer apartado se han analizado los artículos publicados en las revistas en los diferentes años estudiados.

En primer lugar, la revista 3C Empresa presenta un total de 116 artículos publicados en el período establecido. Es importante tener en cuenta que la periodicidad de esta revista en sus inicios era mensual, siendo ya trimestral a partir de su segundo año en circulación. Se aprecia que la cifra contenida en la primera columna va en orden decreciente, siguiendo un proceso de normalización de número de artículos por número de revista, y en consecuencia, por año. Se calcula la media, moda y mediana de documentos por año que en este año es constante, pues la cifra en todos los casos oscila entre 4 y 4,5 .

Tabla 1. Producción científica 3c Empresa.

\begin{tabular}{|l|l|l|l|l|}
\hline Año & No artículos & Media & Moda & Mediana \\
\hline $\mathbf{2 0 1 2}$ & 36 & 4 & 4 & 4 \\
\hline $\mathbf{2 0 1 3}$ & 28 & 4 & 4 & 4 \\
\hline $\mathbf{2 0 1 4}$ & 18 & 4,5 & 4,5 & 4,5 \\
\hline $\mathbf{2 0 1 5}$ & 16 & 4 & 4 & 4 \\
\hline $\mathbf{2 0 1 6}$ & 18 & 4,5 & 4,5 & 4,5 \\
\hline Total & 116 & & & \\
\hline
\end{tabular}

Fuente: elaboración propia.

En segundo lugar, en 3C TIC hay un total de 82 artículos publicados entre 2012 y 2015 . En este caso, a diferencia de $3 C$ Empresa, vemos que la cifra es inferior ya que desde sus inicios 3C TIC comenzó con una periodicidad trimestral. El año 2015 contiene la cifra más alta de artículos en comparación al resto, si bien la diferencia es muy poco significativa. Sin embargo, debe indicarse que esto se debe a que en el primer número de 2015 se publican 8 artículos, pues se trata de una edición especial. En este análisis hemos decidido contabilizarlo porque se cuenta como el número 1, ya que este número no existe y directamente en las ediciones se pasa de la edición especial al número 2 de la revista. En cuanto al análisis de media, moda y mediana también resultan unas cifras uniformes. 
Tabla 2. Producción científica 3c TIC.

\begin{tabular}{|l|l|l|l|l|}
\hline Año & No artículos & Media & Moda & Mediana \\
\hline $\mathbf{2 0 1 2}$ & 12 & 4 & 4 & 4 \\
\hline $\mathbf{2 0 1 3}$ & 16 & 4 & 4 & 4 \\
\hline $\mathbf{2 0 1 4}$ & 16 & 4 & 4 & 4 \\
\hline $\mathbf{2 0 1 5}$ & 20 & 5 & 4 & 4 \\
\hline $\mathbf{2 0 1 6}$ & 18 & 4,5 & 4,5 & 4,5 \\
\hline Total & 82 & & & \\
\hline
\end{tabular}

Fuente: elaboración propia.

Para finalizar, la revista 3c Tecnología presenta un total de 84 artículos publicados en este periodo. Tiene una periodicidad bastante estable, si bien no llega a ser hasta el 2016 cuando termina de normalizarse. La cantidad de artículos por año oscila entre 12 y 22 trabajos por año, lo cual se debe a que en el primer año se edita la revista una vez, mientras que en 2014 cinco. En la misma línea que 3C Empresa y 3 C TIC, los datos referentes a la media, moda y mediana también son bastante uniformes.

Tabla 3. Producción científica 3c Tecnología

\begin{tabular}{|l|l|l|l|l|}
\hline Año & No artículos & Media & Moda & Mediana \\
\hline $\mathbf{2 0 1 2}$ & 12 & 4 & 4 & 4 \\
\hline $\mathbf{2 0 1 3}$ & 16 & 4 & 4 & 4 \\
\hline $\mathbf{2 0 1 4}$ & 22 & 4,4 & 4 & 4 \\
\hline $\mathbf{2 0 1 5}$ & 16 & 54 & 4 & 4 \\
\hline $\mathbf{2 0 1 6}$ & 18 & 4,5 & 4,5 & 4,5 \\
\hline Total & 84 & & & \\
\hline
\end{tabular}

Fuente: elaboración propia.

\subsection{AUTORÍA}

En el siguiente apartado se va a analizar la autoría desde diversas perspectivas. Se desvelan el total de autores por revista y también se indicará el número de autores nacionales e internacionales en el periodo estudiado. Además, se profundizará indicando la procedencia geográfica de los autores de cada revista.

\section{- Número de autores por revista}

Seguidamente se puede apreciar que este indicador es muy semejante en las tres publicaciones. Se parte de un total de 350 autores que han aportado uno o más trabajos a las revistas analizadas. De estos autores, 119 publican en 3C Empresa, 113 en 3C TIC, y finalmente, 3C Tecnología alberga 118 autores. Es destacable su similitud, pues las tres revistas presentan cifras muy parecidas, lo cual se debe a que el número de autores por artículo en las revistas está limitado a cuatro, de modo que en los artículos siempre se oscila entre 1 y 4 autores, por lo que las diferencias en los totales de autores por revistas era de esperar que no serían muy notables. 
Tabla 4. Número de autores por revista.

\begin{tabular}{|l|l|}
\hline Revista & Número autores \\
\hline 3c Empresa & 119 \\
\hline 3c Tic & 113 \\
\hline 3c Tecnología & 118 \\
\hline Total autores & 350 \\
\hline
\end{tabular}

Fuente: elaboración propia.

\section{- Procedencia de los autores}

Por lo que respecta 3 Empresa, el país con autores que más aportan a la revista es España, con un total de 70 autores. Muy por debajo se sitúan Ecuador (19) y México (16), y les siguen Cuba y Puerto Rico con 6 autores cada uno. Finalmente, se encuentran República Dominicana y Venezuela con 1 aportación de cada país. Con lo cual, se concluye que en $3 \mathrm{C}$ Empresa publican un $58,80 \%$ (70) autores nacionales mientras que un $41,10 \%$ (49) internacionales.

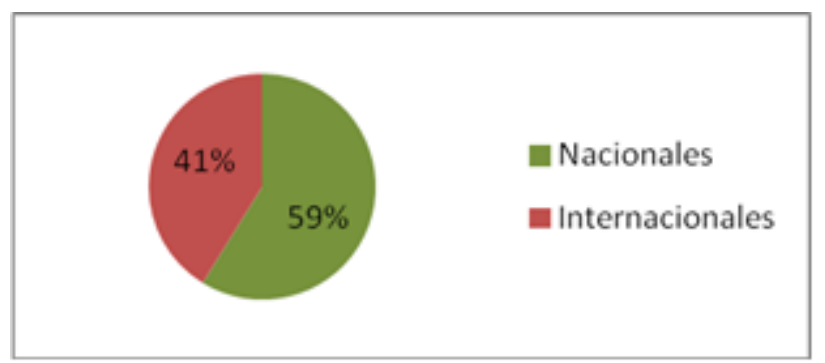

Gráfico 1. Porcentaje de autores nacionales e internacionales en 3C Empresa. Fuente: elaboración propia.

Tabla 5. País de procedencia de los autores en 3C Empresa.

\begin{tabular}{|l|l|}
\hline País & Número autores \\
\hline Cuba & 6 \\
\hline Ecuador & 19 \\
\hline España & 70 \\
\hline México & 16 \\
\hline Puerto Rico & 6 \\
\hline República Dominicana & 1 \\
\hline Venezuela & 1 \\
\hline Total & 119 \\
\hline
\end{tabular}

Fuente: elaboración propia.

En 3C TIC, los autores que más aportaciones realizan también son los españoles, con un total de 62 autores. Bastante por debajo se encuentran autores cuya procedencia es Cuba (26), Ecuador (10), México (8) y Colombia (6), y con tan solo 1 aportación Perú. Por lo tanto, estos datos concluyen que el $54,90 \%$ (62) de autores son nacionales mientras que el $45,10 \%$ (51) son internacionales. 


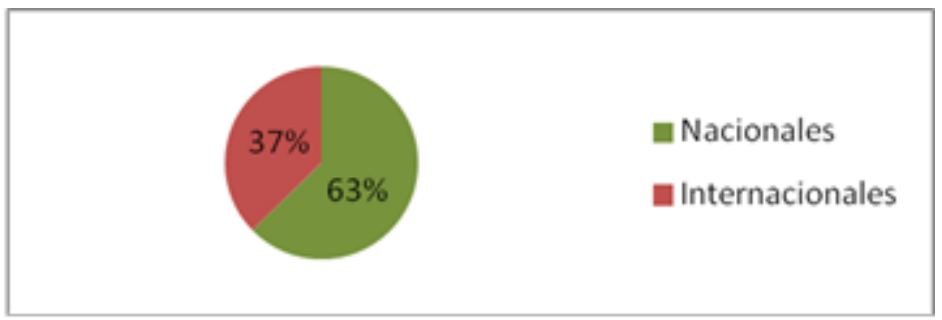

Gráfico 2. Porcentaje de autores nacionales e internacionales en 3C TIC. Fuente: elaboración propia.

Tabla 6. País de procedencia de los autores en 3C TIC.

\begin{tabular}{|l|l|}
\hline País & Número autores \\
\hline Colombia & 6 \\
\hline Cuba & 26 \\
\hline Ecuador & 10 \\
\hline España & 62 \\
\hline México & 8 \\
\hline Perú & 1 \\
\hline Total & 113 \\
\hline
\end{tabular}

Fuente: elaboración propia.

Finalmente, 3C Tecnología constituye la revista con más aportaciones de autores de España, con 74, cifra ligeramente superior a las anteriores. Además de España, la revista también se nutre de aportaciones de autores de Ecuador (26), Cuba (13), Argentina (4), y Portugal (1). Esta revista tiene por tanto el mayor porcentaje de autores nacionales $(62,70 \%)(74)$ frente a los internacionales $(37,30 \%)(44)$ de las tres revistas.

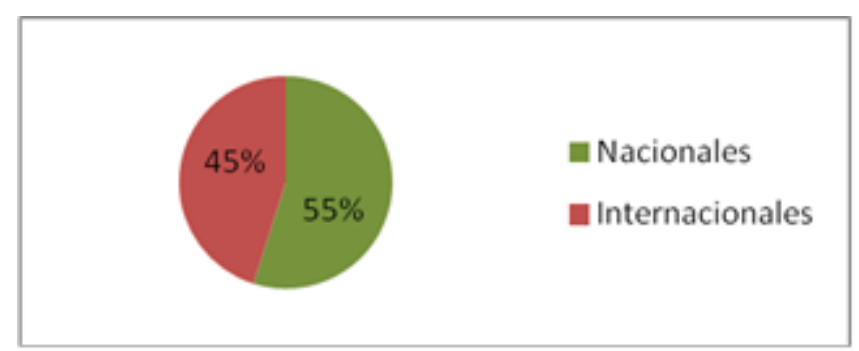

Gráfico 3. Porcentaje de autores nacionales e internacionales en 3C Tecnología.

Fuente: elaboración propia.

Tabla 7. País de procedencia de los autores en 3C Tecnología.

\begin{tabular}{|l|l|}
\hline País & Número autores \\
\hline Argentina & 4 \\
\hline Cuba & 13 \\
\hline Ecuador & 26 \\
\hline España & 74 \\
\hline Portugal & 1 \\
\hline Total & 118 \\
\hline
\end{tabular}

Fuente: elaboración propia. 


\section{- Ley de Lotka}

Se aplica la Ley de Lotka, también conocida como Ley de Productividad de los autores para demostrar que la distribución del número de autores que publican " $\mathrm{n}$ " trabajos es inversamente proporcional a $\mathrm{n}^{2}$ (González Alcaide, 2012). Es decir, se establece una relación cuantitativa entre los autores y su producción en un determinado tiempo, y en este caso, en una revista.

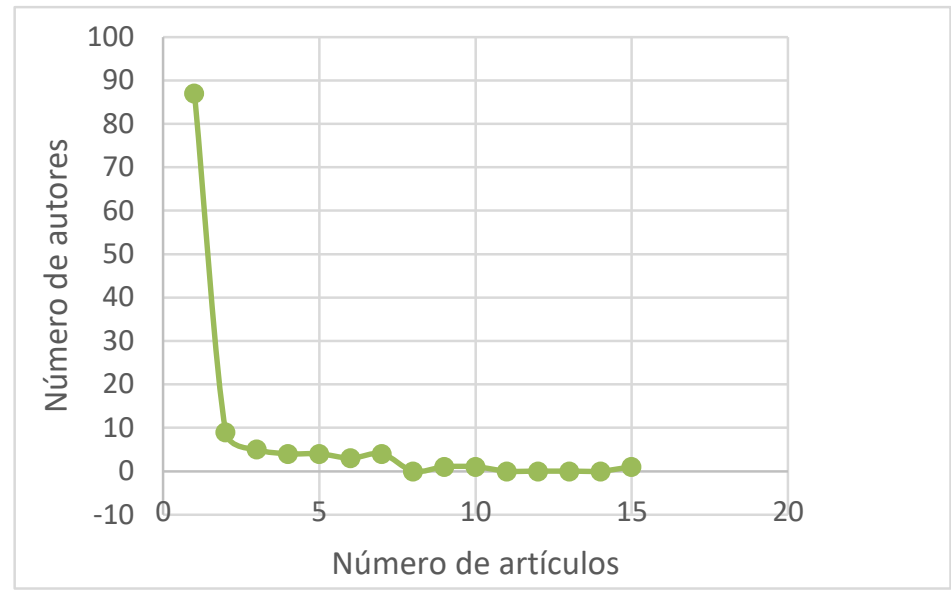

Gráfico 4. Ley de Lotka para 3C Empresa. Fuente: elaboración propia.

En el Gráfico 4 se aprecia una curva exponencial muy notable, pues en primer lugar las cifras indican que hay 87 autores que firman solamente 1 trabajo en $3 C$ Empresa, y esta cifra cae hasta indicar que son 9 autores los que firman 2 trabajos. Los datos se mantienen estables hasta alcanzar las últimas cifras, que revelan que 1 solo autor publica 15 trabajos.

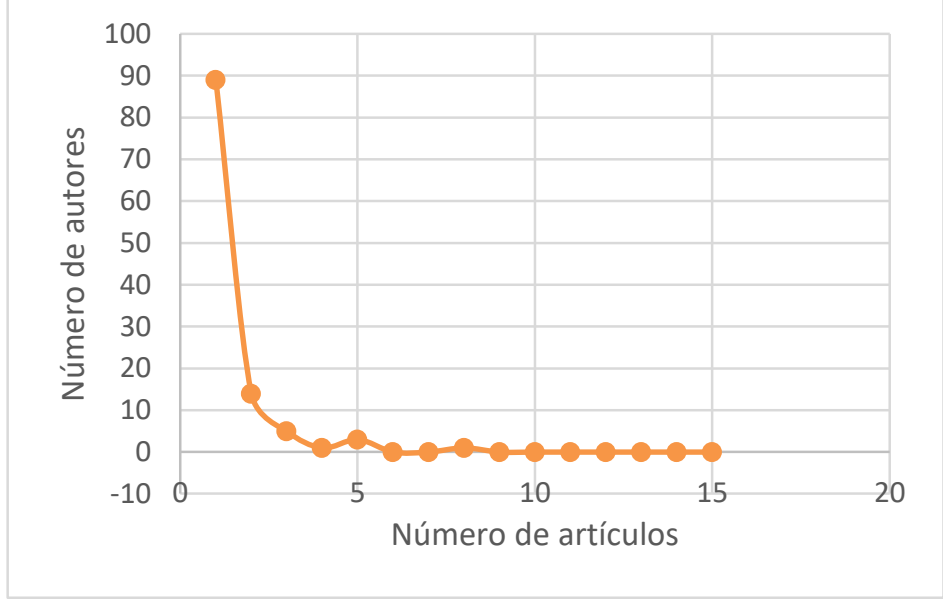

Gráfico 5. Ley de Lotka para 3C TIC. Fuente: elaboración propia.

Los datos para 3C TIC (Gráfico 5) siguen la misma línea que el anterior gráfico. Se aprecia que de nuevo son 89 autores los que firman 1 trabajo, y esta cifra cae a 14 autores, que son los que firman 2 trabajos. Seguidamente observa que son 5 autores los que firman 3 trabajos, hasta llegar a la cifra mínima de 1 autor como firmante de 8 trabajos. 


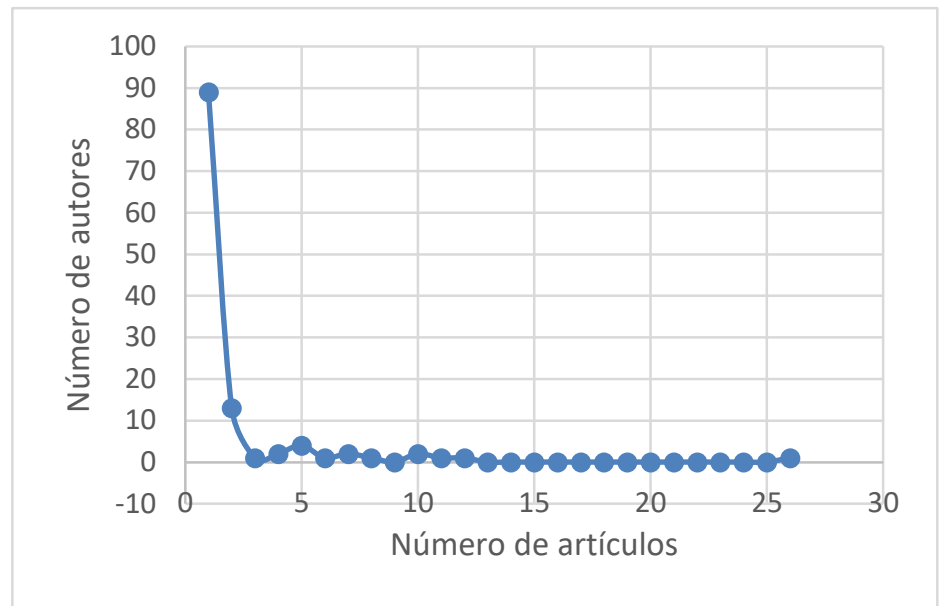

Gráfico 6. Ley de Lotka para 3C Tecnología. Fuente: elaboración propia.

Finalmente, en 3 C Tecnología (Gráfico 6) se parte de que son 89 autores los que firman 1 trabajo, y la siguiente cifra decae hasta 13 los autores firmantes de 2 trabajos. Esta cifra se mantiene más o menos constante hasta 1 autor como firmante de 12 trabajos, y a continuación los datos son inexistentes hasta el caso aislado de 1 autor firmante de 26 trabajos.

Con lo cual, en las tres revistas se cumple la Ley pronunciada por Lotka (1926), pues se halla esta distribución desigual de productividad en los autores independientemente de la revista analizada. Se ha concluido que la mayoría de los autores publican el menor número de trabajos, al mismo tiempo que unos pocos autores publican la mayor parte de los artículos.

\section{CONCLUSIONES}

A modo de conclusión, se puede decir que en cuanto a producción científica, las revistas de la Editorial 3Ciencias han entrado en un proceso de normalización por lo que respecta la cantidad de artículos por número, y en consecuencia, por año. Esto se debe, en primer lugar, a que las revistas, con más de 5 años de trayectoria, se van abriendo un nicho en el sector editorial y dentro de la temática que a cada una le corresponde. En segundo lugar, también influyen en este proceso de normalización las exigencias de las agencias de evaluación e indexaciones en bases de datos, donde cumplir sus criterios de evaluación es indispensable.

Por lo que respecta a la autoría, se aprecia en primer lugar que el número de autores por revista es muy semejante en las tres publicaciones, oscilando el total en todas ellas entre 113 y 119. Por otro lado, predominan autores nacionales, pero cada vez más aumenta la cantidad de autores internacionales, sobretodo de zonas hispanohablantes como México o Ecuador, entre otros.

Finalmente, la Ley de Lotka revela que hay un gran número de autores que aportan una reducida cantidad de producción científica mientras que un reducido grupo de autores publican gran cantidad de trabajos. 


\section{REFERENCIAS BIBLIOGRÁFICAS}

Ardanuy, J. (2012). Breve introducción a la bibliometría. Universitat de Barcelona: Departament de Biblioteconomía i Documentació.

Editorial 3Ciencias. (2017). Recuperado de: https://www.3ciencias.com/.

González Alcaide, G. (2012). Bibliometría. Fundamentos teóricos, aplicaciones y metodología para el análisis de la literatura científico-médica. (Valencia) España: Psylicom.

Hammarfelt, B. y Rushforth, A. (2017). Indicators as judgment devices: An empirical study of citizen bibliometrics in research evaluation. Research Evaluation, 26(3), 169-180. DOI: 10.1093/reseval/rvx018.

Lotka, A.J. (1926). The frequency distribution of scientific productivity. Journal of the Washington Academy of Sciences. 16(12), 317-324.

Paz Enrique, L.E., Peralta González, M.J. y Hernández Alfonso, E.A. (2016). Estudio bibliométrico de la Revista Centro Agrícola, Cuba. E-Ciencias de la Información, 6(2). DOI: https://doi.org/10.15517/eci.v6i2.25273.

Zeleznik, D., Blazun Vosner, H. y Kokol, P. (2017). A bibliometric analysis of the Journal of Advanced Nursing, 1976-2015. Journal of Advanced Nursing, 73(10), 2407-2419. DOI: 10.1111/jan.13296. 